\title{
Stereoselective Block of hERG Channel by Bupivacaine Scrutinized at Molecular Level
}

\author{
Liliana Sintra Griloßab, Pierre-Alain Carrupt ${ }^{\mathrm{a}}$, and Antoine Daina*a \\ §SCS-DSM Nutritional Products Award Winner (Poster Presentation)
}

\begin{abstract}
In the heart, the $\mathrm{hERG}$ voltage-gated potassium channel mediates the $\mathrm{I}_{\mathrm{Kr}}$ current, which is crucial for the duration of cardiac action potential. Undesired block of the channel may prolong the QT interval with increased risk of malignant ventricular arrhythmia called torsades de pointes. Although the molecular determinants of hERG block are intensively studied, stereoselectivity has been poorly investigated. Levo-(S)-bupivacaine was the first drug reported to have higher affinity for hERG than its enantiomer. This study aims at understanding the principles underlying the stereoselectivity of bupivacaine block with the help of molecular modeling. Putative binding modes of levo-(S)- and dextro-(R)-bupivacaine inside an open form model of hERG channel were predicted by docking simulations, allowing a clear depiction of ligand-protein interactions. Estimated binding energies for both enantiomers to wild-type channel are in line with previously published electrophysiology measurements. These results may be considered as a confirmation at the molecular level of bupivacaine stereoselective binding towards hERG. Moreover this information lays the foundations for a structural guideline to filter out potentially cardiotoxic drug candidates in silico.
\end{abstract}

Keywords: Bupivacaine $\cdot$ Cardiotoxicity $\cdot$ Docking $\cdot$ hERG-block $\cdot$ Stereoselectivity

\section{Introduction}

The hERG (human ether a go-go related gene) channel is a voltage-gated potassium channel that plays a crucial role in the repolarization phase of the cardiomyocyte action potential. ${ }^{[1]} \mathrm{KCNH} 2$ gene encodes the $\alpha$-subunit that assembles into a homotetramer to form a functional channel conducting specifically $\mathrm{K}^{+}$ions (Fig. 1A, left). The pore-forming domain of hERG is constituted of the transmembrane helix S5, the pore helix and selectivity filter, and the S6 transmembrane domain (Fig. 1A, right). Many loss-of-function mutations in $\mathrm{KCNH} 2$ gene can prolong the QT interval on the ECG and trigger potentially lethal arrhythmia known as torsades de pointes, which may cause sudden death. ${ }^{[2]}$ Such genetic disorders are referred to as congenital Long QT Syndrome (cLQTS). ${ }^{[3]}$

Numerous structurally diverse drugs, among others antifungal ketoconazole, ${ }^{[4]}$ antipsychotic chlorpromazine, ${ }^{[5]}$ or prokinetic cisaprid, ${ }^{[6]}$ have the ability to unintentionally block the hERG channel leading to the so-called drug-induced Long QT Syndrome (diLQTS). ${ }^{[7]}$ Although molecular determinants of hERG block have been intensively studied, stereoselectivity has been scarcely investigated. ${ }^{[8-11]}$ Levo$(S)$-bupivacaine (Fig. 1B, left), a broadly used anesthetic, was the first drug reported to be more potent than its enantiomer
( 2-fold) to block hERG channels. ${ }^{[9-11]}$ We also focused our interest on another chiral drug, $(R, S)$-terfenadine (Fig. 1B, right). Terfenadine is a well-known highaffinity blocker of hERG, ${ }^{[12,13]}$ that shares similar chemical moieties with bupivacaine. Both structures encompass a piperidine ring $\mathrm{N}$-substituted by an alkyl chain. Moreover one of the terfenadine aromatic ring presents similar distance from the basic nitrogen compared to the $o, o$-dimethylphenyl of the bupivacaine. Terfenadine was employed as an anti-histaminic drug until its adverse effects on hERG ${ }^{[14]}$ and QT prolongation ${ }^{[15]}$ led to its withdrawal from the market. In notable contrast with bupivacaine, stereoselective block of the
A

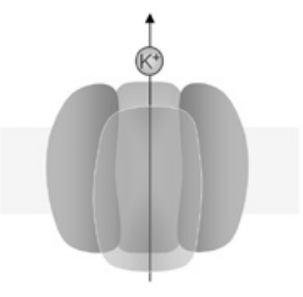

B

(R,S)-bupivacaine

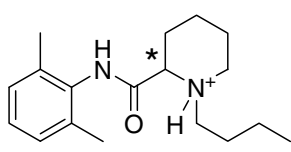

out

in

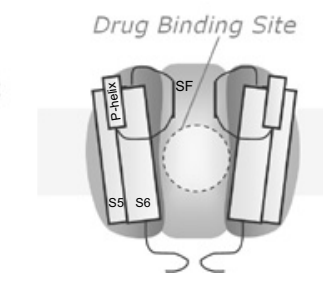
depicting the tetrameric structure of hERG channel, with poreconstituting unit formed by $\mathrm{S} 5$ and S6 transmembrane domains, the porehelix (P-helix) and the selectivity filter (SF). (B) Chemical structures, protonated as at $\mathrm{pH} 7.4$ of $(R, S)$-bupivacaine and $(R, S)$-terfenadine with chiral center marked with an asterisk. 
hERG channel has never been reported for terfenadine.

In the present study, the stereoselective block of the hERG channel by bupivacaine enantiomers was thoroughly investigated by a fine-tuned modeling methodology in order to rationalize the stereoselective binding at the molecular level.

\section{Methods}

\section{Selection and Preparation of the hERG Target Structure}

Different publicly available homology models of the hERG channel were considered and meticulously evaluated as potential targets for the present structure-based studies. One of the published models provided by Farid et al., ${ }^{[16]}$ based on the bacterial KvAP crystal (pdb entry:1orq), was finally selected for two main reasons: i) the open conformation of the channel appears accessible for the binding of ligands and ii) it was built with the ligand $(S)$-terfenadine already present inside using the so-called induced-fit docking protocol which involves successive steps of Glide docking and Prime protein modeling. ${ }^{[17]}$ Moreover, Farid and colleagues performed Glide 3.0 docking (Schrödinger, LLC, Portland, OR) towards this structure for a set of known blockers with well-characterized effects on hERG. Interestingly their simulations predicted comparable binding modes for terfenadine, cisapride, sertindole, ibutilide and clofilium, adding confidence to the docking solutions obtained. For our needs, all hydrogens were added to the protein residues of the model by the procedure embedded in the BIOPOLYMER module of Sybyl 8.0 (Tripos Associates, Inc., StLouis, MO), $(S)$-terfenadine was removed and the remaining hERG structure was used as target for docking without further refinement or optimization.

\section{Preparation of Terfenadine and Bupivacaine Ligand Structures}

Both enantiomers of terfenadine, i.e. 1-(4-tert-butylphenyl)-4-[4-[hydroxyldi(phenyl)methyl]piperidin-1-yl]butan-1ol, and both enantiomers of bupivacaine, i.e. 1-butyl-N-(2,6-dimethylphenyl)piperidine-2-carboxamide (Fig. 1B) were modeled with a protonated piperidine nitrogen, Gasteiger and Marsili partial atomic charges and a formal charge of +1 within the Sybyl 8.0 environment. ${ }^{[18]}$ A first energy minimization was performed in the Tripos force field, but the flexibility of the piperidine rings stressed the need for further conformational search. The cationic species were submitted to a Monte Carlo (MC) conformational analysis according to the semi-empirical AM1 molecular orbital theory as implemented in Spartan '06
(Wavefunction Inc, Irvine, CA). The selected conformations were then optimized at HF/3-21G level of theory using Spartan '06 and, for every compound, the lowest energy conformer was used as ligand input for docking towards hERG.

\section{Docking}

Automated molecular docking was carried out using the GOLD program version 4.0 (Gold Suite, CCDC Software Ltd., Cambridge, UK). The binding site was defined following the definition of Stansfeld et al., ${ }^{[19]}$ namely as all protein atoms within $20 \AA$ from the natural $\mathrm{K}^{+}$ion $\mathrm{S}$. This ion is not present in the structure, but coordinates were retrieved from backbone overlay of the selectivity filter of KcsA crystallized subunit including $\mathrm{K}^{+}$ions (pdb entry: $1 \mathrm{k} 4 \mathrm{c}$ ) and our target structure. Coordinates of potassium ion K3005 correspond to $\mathrm{S}_{\text {cav }}$. Three independent docking simulations, each asking for 20 solutions per ligand, were run in parallel, according to the GOLD genetic algorithm default parameters. Ligands were considered as flexible and protein as rigid, except all hydroxyl and amine moieties. Moreover, the Tyr652 side-chain - crucial for binding as well as very keen to adapt its orientation was considered as fully flexible according to the rotamer library available in GOLD 4.0. In order to limit the complexity of solution analyses due to the rotational symmetry of the tetramer, a slight constraint favoring hydrogen-bonding with Ser624 of one specific subunit was applied, in accordance with Farid's observations.

The 60 docking solutions per ligand were evaluated by the GoldScore function with default parameters. Further criteria were employed to select one or a few more docking poses to be submitted to post-docking treatment: i) the population of clusters of solutions based on rmsd on heavy atoms ii) careful visual inspection focused on intermolecular interactions involving a priori important pharmacophoric features such as, in particular, the protonated nitrogen atom and the aromatic rings.

\section{Molecular Mechanics Post-docking Optimization and Binding Affinity Prediction}

The best solutions according to the above-mentioned criteria and their corresponding protein structures were then submitted to molecular mechanics treatment within the AMBER 10 environment. ${ }^{[20]}$ This implies the traditional all-atom AMBER force field for the protein atoms, the GAFF force field and semi-empirical AM1BCC charges for the ligand atoms, together with implicit solvation model terms. This procedure, adapted from Graves et al. .21] $^{\text {[1 }}$ consists of three main steps where, in our case, only the ligand is allowed to move inside the hERG channel kept rigid: i) a 100 steps minimization with a conjugate gradient method; ii) a Langevin molecular dynamic of 3000 steps at constant temperature of $300 \mathrm{~K}$; iii) a second minimization identical to i). The procedure, beside geometry optimization and thus refined binding mode prediction, leads to an approximation of the affinity of the ligand for the hERG channel by estimating the free energy of binding $(\Delta \mathrm{G})$, according to Eqn. (1):

$\Delta \mathrm{G}=\mathrm{E}_{\text {binding }}=\mathrm{E}_{\text {complex }}-\left(\mathrm{E}_{\text {protein }}+\mathrm{E}_{\text {ligand }}\right)$

where $\mathrm{E}_{\text {complex }}, \mathrm{E}_{\text {protein }}, \mathrm{E}_{\text {ligand }}$ are the solvated internal energy of the complex, the protein and the ligand, respectively, as computed by the force fields enriched by implicit solvation terms according to the GB/SA model developed by Onufriev et al. ${ }^{[22]}$ and the LCPO algorithm term to account for the surface area.[23]

\section{Analysis of Binding Mode and Interactions}

Geometries and intermolecular interactions within the optimized virtual complexes were finally analyzed visually and with the help of Maestro 8.5 measurement tools (Schrödinger, LLC, Portland, OR), where good contacts are defined with a cutoff ratio of $1.3 \AA$. Final solutions for all enantiomers were superimposed in the same coordinate system for comparison of binding modes and location of similar pharmacophoric features.

\section{Results}

\section{Binding Modes of (R)- and (S)-Terfenadine}

The redocking of $(S)$-terfenadine in the homology model, followed by force field optimization of the ligand inside the binding site, was used to calibrate the setup of the entire simulation. Parameters leading to the best overlap of selected $(S)$-terfenadine solutions and the native pose adopted in Farid's model were retained and described above. Our $(S)$-terfenadine predicted binding mode and the ligand as found in the published homology model are presented in Fig. 2A and 2C, respectively. As terfenadine was never reported as a stereoselective blocker of hERG, we performed the docking of the $(R)$-terfenadine with the same modeling protocol. Interestingly, the $(R)$ terfenadine binding mode (Fig. $2 \mathrm{~B}$ ) is very similar to that of the $(S)$-enantiomer. Location of the chiral center allows only minor conformational and positional changes for the $(R)$ - and $(S)$-enantiomers, resulting globally in the flip of the hydroxyl group. For $(S)$-terfenadine, this group acts as a hydrogen donor for the oxygen of Ser649 hy- 


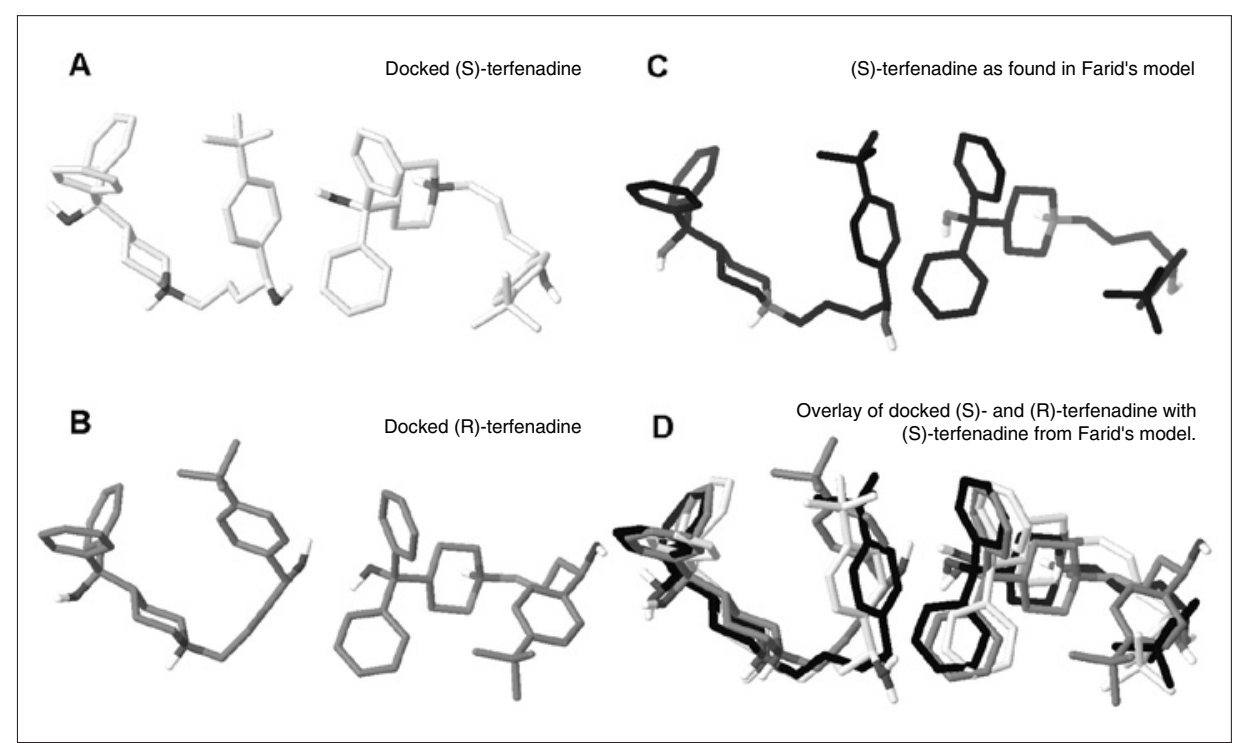

Fig. 2. Binding modes from two orthogonal views of enantiomers (S)-terfenadine (carbons in light grey; Panel A) and (R)-terfenadine (carbons in medium grey; Panel B) as predicted by our docking methodology compared with (S)-terfenadine from Farid's homology model[16] (carbon on black; Panel C). Panel D shows superimposed binding modes for terfenadine enantiomers with an excellent overlap of all poses (same color-coding). All protein atoms and non-polar hydrogens of terfenadine are omitted for clarity.

Table 1. Calculated Gibbs free energy of binding ( $\Delta \mathrm{G}, \mathrm{kcal} / \mathrm{mol})$ within the AMBER 10 environment including implicit solvation and surface terms for the different ligands and estimated binding energy difference $\left(\Delta \mathrm{G}_{(\mathrm{R}) \text {-form }}-\Delta \mathrm{G}_{(\mathrm{S}) \text {-form }}=\Delta(\Delta \mathrm{G}), \mathrm{kcal} / \mathrm{mol}\right)$.

$\begin{array}{lll}\text { Ligand } & (\Delta \mathbf{G}), \mathbf{k c a l} / \mathbf{m o l} & \Delta(\Delta \mathbf{G}), \mathbf{k c a l} / \mathbf{m o l} \\ (R) \text {-terfenadine } & -26.73 & \\ (S) \text {-terfenadine } & -26.03 & -0.70(R)=(S) \\ \text { dextro-(R)-bupivacaine } & -17.14 & \\ \text { levo-(S)-bupivacaine } & -20.85 & 3.71(R)>(S) \\ \end{array}$

droxyl side-chain to form a H-bond. In the case of $(R)$-terfenadine, orientation of the hydroxyl group does not allow H-bond interaction. The tert-butylphenyl end undergoes a $90^{\circ}$ rotation, yet keeping its general location in the hERG binding site, thus still involved in aromatic-aromatic interactions with residue phenylalanine in position 656. As expected by the constraint, for both enantiomers Ser624 is involved in electrostatic interaction with basic nitrogen. An equivalent serine on another $\alpha$-subunit is involved in hydrogen bond interaction with the hydroxyl group at the benzylic position of $(R)$ - and $(S)$-terfenadine. The remarkable common binding mode predicted for both enantiomers (Fig. 2D) lets us assume there is no stereoselectivity for the binding of terfenadine within the pore of the channel.

Estimated free energies of binding $(\Delta \mathrm{G}$, Table 1) were calculated during optimization of ligands inside the binding site of hERG in AMBER environment using an implicit solvent model (GB/SA). For terfenadine enantiomers, $(R)$ - and $(S)$-forms present similarfree energies of binding, with a difference $\left(\Delta(\Delta \mathrm{G})=\Delta \mathrm{G}_{(R) \text {-form }}-\Delta \mathrm{G}_{(S) \text {-form }}\right)$ lesser than $1 \mathrm{kcal} / \mathrm{mol}$ and regarded as negligible. These results also claim for the same binding affinity of terfenadine enantiomers for hERG open-channel.

\section{Binding Modes of Dextro-(R)- and Levo-(S)-bupivacaine}

After validation of the modeling strategy with terfenadine, the same methodology was followed for dextro- $(R)$ - and levo- $(S)$-bupivacaine docking toward the binding site of hERG channel. Final optimized binding modes of bupivacaine were analyzed considering only residues of the pore with atoms at $5 \AA$ distance from the ligands. The main striking difference in the binding modes of the enantiomers is the opposing orientation of the butyl chain. It is worth mentioning that the basic nitrogen of the piperidine ring as well as the carbonyl group of the amide are pointing towards the top of the hERG cavity and occupy the same position in the binding site. Good contacts between the ligand and the protein (dashed lines, Fig. 3) were elicited by Maestro measurement tools, which highlighted residues, such as Leu622, Thr623, Ser624, Ser649, Tyr652, Ala653 and Phe656, involved in the drug recognition. Among these, Tyr652 and Ser624 are the residues that display the highest number of good contacts and involve simultaneously three to four subunits of the channel (Fig. 3). Both bupivacaine enantiomers reveal electrostatic interactions between the carbonyl of the amide and Ser624 of multiple $\alpha$ subunits. In the final optimized binding mode, levo-( $S)$-bupivacaine calls for the contribution of the tyrosine residues in position 652 of all $\alpha$-subunits. Conversely, dextro- $(R)$-bupivacaine interacts with only three tyrosine residues. Moreover the $o, o$-dimethylphenyl group creates, for both enantiomers, numerous good contacts with Tyr652 of $\alpha$-subunit I (Fig. 3), thus keeping the aromatic moiety of the ligand in a same favorable area. The number of $\alpha$-subunits involved in interactions with the ligand is also different regarding Phe656, which is the second aromatic residue protruding in the inner cavity. Benzyl side-chains of two $\alpha$-subunits (III and IV, Fig. 3A) are involved in good contacts with levo- $(S)$-bupivacaine, whereas for dextro- $(R)$-bupivacaine only one $\alpha$-subunit (III, Fig. 3B) slightly contributes to interactions with the ligand. The difference in the molecular recognition with additional contribution of two aromatic residues could be a clue to explain the higher affinity of block described experimentally for levo-(S)-bupivacaine. ${ }^{[9-11]}$ Moreover and in contrast to the terfenadine estimations, free energy of binding calculations predict the levo- $(S)$-bupivacaine-hERG complex to be more stable than the dextro- $(R)$-bupivacaine-hERG complex by nearly $4 \mathrm{kcal} / \mathrm{mol}$. These results reveal a stereoselective behavior in the binding of bupivacaine within the $\mathrm{K}^{+}$channel, with levo- $(S)$-form showing higher affinity than the dextro- $(R)$-bupivacaine.

\section{Discussion}

Here we present a molecular investigation of hERG stereoselective block by bupivacaine enantiomers. The computational strategy was first validated by redocking $(S)$-terfenadine enantiomer toward the described homology model. The difficulty of this particular docking case laid in the symmetrical nature of the ligands together with 


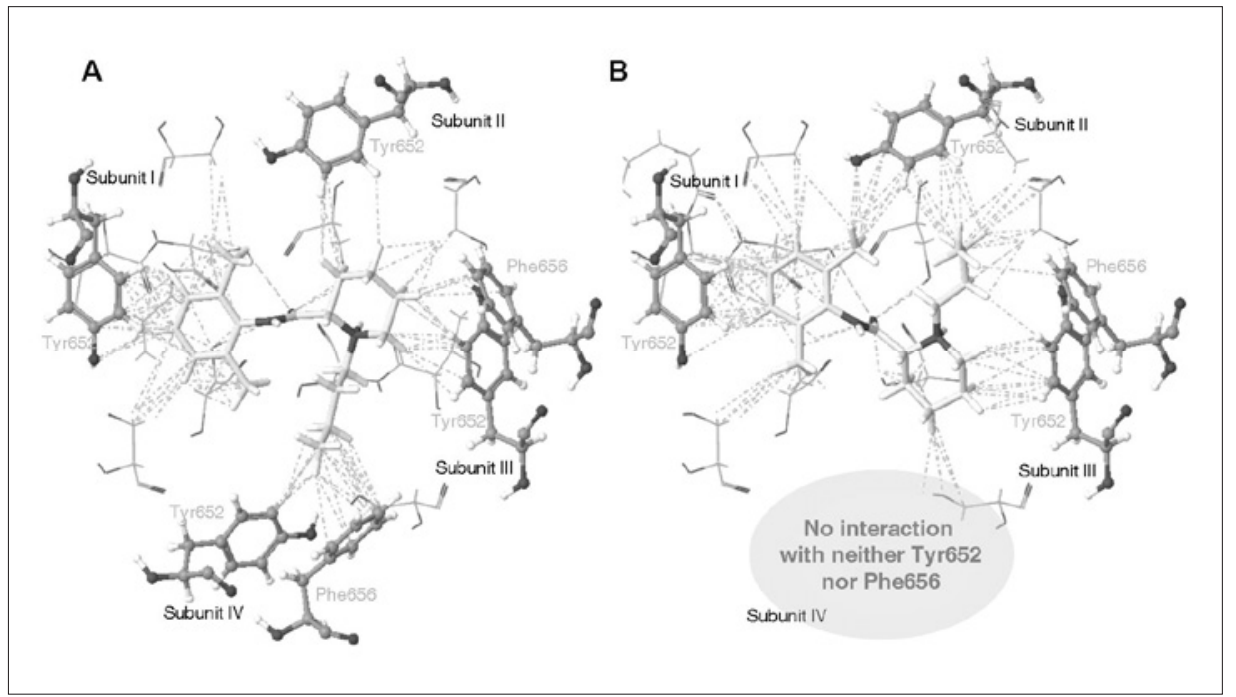

Fig. 3. (A) Dextro-(R)-bupivacaine and (B) levo-(S)-bupivacaine (in stick representation with carbons in light grey), and hERG residues (carbons in dark grey) creating good contacts (dashed lines) with the ligand within a $5 \AA$ shell around it (Maestro 8.5). Both (A) and (B) are seen from the same viewpoint, i.e. from the inner side of the channel cavity. Tyr652 residues have been flexibilized during docking process (GOLD 4.0). Known important residues (Tyr652 and Phe656) are highlighted with ball-and-stick representation, others are in wireframe.

the tetrameric structure of hERG channel, which introduces a rotational symmetry. The multiplication of identical binding modes resulting from the symmetry of the channel was overcome by adding a slight constraint favoring hydrogen-bond formation with one Ser624 residue. The predicted binding mode of the $(S)$-terfenadine is very similar to the one published by Farid et al., though obtained by a totally different approach. ${ }^{[16]}$ In their previous work, $(S)$-terfenadine blocker was shown to be in close contact with four Tyr652 and two Phe656 residues, and the nature of $\pi-\pi$ interactions with the aromatic groups of the ligand was explicitly specified. For both $(R)$ - or $(S)$-terfenadine, two of the four tyrosine sidechains display T-shaped (edge-to-face) $\pi-\pi$ interactions with phenyl groups of the ligands. ${ }^{[16]}$ Regarding phenylalanine residues, they reported two T-shaped interactions for $(R)$-terfenadine, whereas the $(S)$-enantiomer made one T-shaped and one parallel displaced stacking. ${ }^{[16]}$ The binding mode of $(R)$-terfenadine was not published by Farid and colleagues, but our final binding solution offers an explanation to this difference. As mentioned before, one of the few changes observed in the docking solutions for the anti-histaminic enantiomers is the orientation of the tert-butylphenyl group. Undergoing a $90^{\circ}$ rotation switches the aromatic interaction from T-shaped to parallel displaced mode. These kind of aromatic interactions are known to be isoenergetic and, as such, equally stabilizing for molecular recognition. ${ }^{[24]}$ However, the parallel displaced interaction between one aromatic ring of the docked $(S)$-terfenadine and the side-chain of Phe656 appears to be far from optimal in terms of distance and parallelism. This loss of stabilization is counterbalanced by the additional hydrogen-bond between the tertiary alcohol and Ser649 side-chain predicted to be only displayed by $(S)$ terfenadine and not $(R)$-terfenadine. With the exception of these two points, both enantiomers present very similar binding modes suggesting overall similar interactions with the channel. Calculations of free energy of binding provided supplementary evidence for equal affinity of terfenadine enantiomers to hERG binding site, which is in agreement with the absence of any reported stereoselective block. molecule established to block more potently the hERG channel with its levo- $(S)$ form than its racemate. ${ }^{[9-11]}$ Levo- $(S)$-bupivacaine was commercialized because it was demonstrated to be safer for local anesthesia than the racemic mixture. ${ }^{[25]}$ The purpose of this work was not to reconsider the extensive use of this enantiomer in anesthesiology, but to investigate the molecular determinants of hERG stereoselective block. Whereas terfenadine enantiomers presented similar binding modes, final docking solutions of bupivacaine $(R)$ - and $(S)$-forms differed. Importantly, the opposing orientation of the butyl rest allows levo- $(S)$-bupivacaine to interact with two additional aromatic side-chains, Tyr652 and Phe656 of the fourth $\alpha$-subunit, already known to be important for hERG block by local anesthetics.[10] This observed difference in the molecular recognition could explain the higher blocking capability of levo- $(S)$-bupivacaine displayed at electro-
In contrast, bupivacaine was the first physiologcal level, if one assumes that binding of the molecules inside the pore prevents normal $\mathrm{K}^{+}$ion movement and so blocks the hERG current. The stereodependant binding mode is corroborated by the estimation of a more favorable free energy of binding for the levo- $(S)$-form. Moreover, Tyr652 and Phe656 aromatic residues have already been mentioned by Farid et al. for their influential $\pi-\pi$ interactions with terfenadine. ${ }^{[16]}$ For both enantiomers of bupivacaine, Tyr652 of the $\alpha$-subunit I (Fig. 3) might be involved in parallel displaced $\pi-\pi$ interactions with the $o, o$-dimethyphenyl ring however, the distance and angle between the two aromatic plans are not the most favorable for stabilization.

The propensity of numerous structurally diverse drugs to block the hERG channel is, at least in part, due to its unusually large cavity. In contrast to other voltagegated $\mathrm{K}^{+}$channels, hERG lacks a PxP-kink motif in the S6 helices that would restrict the inner cavity size. ${ }^{[26]}$ It is noteworthy that most of Farid's docked ligands as well as our bupivacaine solutions are located in the same region of the large area explored by the engine. Predicted binding modes are positioned just beneath the selectivity filter at the very top of the vestibule. Superimposition of the terfenadine and bupivacaine binding modes highlights some pharmacophoric features previously described:[27-29] aromatic rings (o,o-dimethylphenyl group of bupivacaine and one phenyl ring of terfenadine) as well as the basic piperidine nitrogen show remarkable overlap and appear fundamental for $\mathrm{hERG}$ channel to recognize these blockers.

The location of the binding site observed in the present case is due to the presence of another key structural determinant of hERG: the aromatic residues Tyr652 and Phe656 that protrude in the pore and that were identified as important partners for interaction with our ligands. Such residues are common to hERG and the related ether à go-go (EAG) channels, but again not to other voltage-gated channels. [30] Mutation of one of these residues into alanine has first been reported to dramatically affect block of hERG by MK-499, a methanesulfonanilide antiarrhythmic drug, ${ }^{[31]}$ and has been observed later for many compounds, ${ }^{[31-34]}$ including terfenadine and local anesthetics. ${ }^{[10]}$ The crucial role of these aromatic amino acids has long been attributed to formation of $\pi$ stacking interactions with aromatic rings and $\pi$-cation interactions with the basic group of the ligand. Farid ${ }^{[16]}$ and Zachariae ${ }^{[35]}$ assign the importance of these amino acids to their concentric arrangement in the cavity allowing multiple $\pi$-stacking and/or hydrophobic interactions with various combinations of Tyr652 and Phe656 
side-chains. The same observations about aromatic interactions were made for bupivacaine enantiomers. In Farid's proposed model, it is suggested that polar groups, including the highly prevalent basic nitrogen, tend to localize in a hydrophilic volume associated with Ser624. ${ }^{[16]}$ Importance of this residue for binding was confirmed by Kamiya et al., ${ }^{[36]}$ when mutation of serine to alanine in position 624 significantly decreased terfenadine block of hERG. Surprisingly, no evident hydrogen bond was retrieved in our final optimized pose between the basic nitrogen and oxygen of Ser624 as seen for terfenadine, even though this docking solution would be favored by the constraint. The explanation lays in the geometry of bupivacaine. The predicted bioactive conformation is very close to an energy minimum and displays an intramolecular interaction between the protonated piperidine nitrogen and one electronic lone pair on the carbonyl oxygen of the amide. The H-bond donor capacity is consumed by this internal nonbonded force. However, careful inspection of the reformed complexes revealed probable electrostatic interactions between the carbonyl group and residues Ser624. The second lone pair, not involved in the intramolecular interaction, is still able to contribute significantly to intermolecular recognition inside the hydrophilic volume defined by Farid and coworkers.

\section{Conclusion and Perspectives}

This target-based study involving multiple symmetries is aimed at understanding the principles underlying the stereoselectivity of hERG block by bupivacaine. To achieve this goal, we validated our computational strategy by redocking $(S)$-terfenadine ligand in a carefully selected published homology model. We were able to reproduce, using a workflow of different modeling techniques, the binding pose obtained by Farid et al. Previous conclusions about the absence of stereospecific block of hERG by terfenadine were confirmed by predicting similar binding modes for both enantiomers and no significant difference in the free energy of binding. Interestingly, docking and molecular mechanics post-docking processing of bupivacaine enantiomers corroborated the stereoselective behavior towards the hERG channel. In the latter case, predicted binding modes and estimated free energies of binding indicated higher affinity of levo- $(S)$-bupivacaine for the $\mathrm{K}^{+}$channel binding site. Moreover, our study emphasized the role of aromatic amino acids Tyr652 and Phe656 lining the channel cavity in their $\pi-\pi$ interactions with the ligand as well as the electrostatic interactions in the hydrophilic volume at the intracellular base of the selectivity filter associated with Ser624.

The complex case of enantiomeric block reproduced at a molecular level gives confidence to the strategy applied. Nevertheless, a more detailed understanding of the structural basis of binding would be gained by docking structurally-related molecules or simulations toward mutated hERG structures for the sake of comparison with experimental results. Furthermore, the validity domain of this model may appear to be narrowed to the structure of bupivacaine, terfenadine and possibly to chemicals sharing a similar pharmacophore. Since the hERG channel is known to recognize an extremely large variety of compounds, it is imperative to challenge our predictions with known blockers showing significant structural variations.

In this perspective, we believe this work lays robust foundations for a structure-based design approach to overcome or even anticipate serious cardiotoxic issues faced by many medicinal chemistry projects.

Received: January 5, 2010

[1] M. C. Sanguinetti, C. G. Jiang, M. E. Curran, M. T. Keating, Cell 1995, 81, 299.

[2] M. C. Sanguinetti, M. Tristani-Firouzi, Nature 2006, 440, 463.

[3] W. Shimizu, Cardiovasc. Res. 2005, 67, 347

[4] R. Dumaine, M. L. Roy, A. M. Brown, J. Pharmacol. Exp. Ther. 1998, 286, 727.

[5] D. Thomas, K. Wu, S. Kathöfer, H. A. Katus, W. Schoels, J. Kiehn, C. A. Karle, Brit. J. Pharmacol. 2003, 139, 567.

[6] S. Mohammad, Z. Zhou, Q. Gong, C. T. January, Am. J. Physiol. 1997, 273, H2534.

[7] D. M. Roden, N. Engl. J. Med. 2004, 350, 1013.

[8] C. B. Eap, S. Crettol, J. S. Rougier, J. Schläpfer, L. Sintra Grilo, J. J. Déglon, J. Besson, M. Croquette-Krokar, P. A. Carrupt, H. Abriel, Clin. Pharmacol. Ther. 2007, 81, 719.

[9] T. Gonzalez, C. Arias, R. Caballero, I. Moreno, E. Delpon, J. Tamargo, C. Valenzuela, Brit. J. Pharmacol. 2002, 137, 1269.

[10] C. C. Siebrands, N. Schmitt, P. Friederich, Anesthesiology 2005, 103, 102.

[11] P. Friederich, A. Solth, S. Schillemeit, D. Isbrandt, Brit. J. Anaesth. 2004, 92, 93.

[12] D. Rampe, M. L. Roy, A. Dennis, A. M. Brown, FEBS Lett. 1997, 417, 28.

[13] W. J. Crumb, J. Pharmacol. Exp. Ther. 2000, 292, 261

[14] M. Roy, R. Dumaine, A. M. Brown, Circulation 1996, $94,817$.

[15] S. P. Pinney, B. S. Koller, M. R. Franz, R. L. Woosley, J. Cardiovasc. Pharmacol. 1995, 25, 30

[16] R. Farid, T. Day, R. A. Friesner, R. A. Pearlstein, Bioorg. Med. Chem. 2006, 14, 3160.

[17] W. Sherman, T. Day, M. P. Jacobson, R. A. Friesner, R. Farid, J. Med. Chem. 2006, 49, 534.

[18] J. Gasteiger, M. Marsili, Tetrahedron 1980, 36, 3219.

[19] P. J. Stansfeld, P. Gedeck, M. Gosling, B. Cox, J. S. Mitcheson, M. J. Sutcliffe, Proteins: Struct. Funct. Bioinf. 2007, 68, 568.

[20] AMBER 10, D. A. Case, T. A. Darden, T. E. Cheatham, C. L. Simmerling, J. Wang, R. E. Duke, R. Luo, M. Crowley, R. C. Walker,
W. Zhang, M. Merz, B. Wang, S. Hayik, G. Roitberg, G. Seabra, I. Kolossváry, K. F. Wong, F. Paesani, J. Vanicek, X. Wu, S. R. Brozell, T. Steinbrecher, H. Gohlke, L. Yang, C. Tan, J. Mongan, V. Hornak, G. Cui, D. H. Mathews, M G. Seetin, C. Sagui, V. Babin, P. A. Kollman, University of California, San Francisco, 2008.

[21] A. P. Graves, D. M. Shivakumar, S. E. Boyce, M. P. Jacobson, D. A. Case, B. K. Shoichet, J. Mol. Biol. 2008, 377, 914 .

[22] A. Onufriev, D. Bashford, D. A. Case, Proteins: Struct. Funct. Bioinf. 2004, 55, 383.

[23] J. Weiser, P. S. Shenkin, W. C. Still, J. Comput. Chem. 1999, 20, 217

[24] P. Hobza, H. L. Selzle, E. W. Schlag, J. Phys. Chem. 1996, 100, 18790

[25] H. Bardsley, R. Gristwood, H. Baker, N. Watson, W. Nimmo, Br. J. Clin. Pharmacol. 1998, 46, 245.

[26] J. S. Mitcheson, Br. J. Pharmacol. 2003, 139, 883.

[27] J. Morgan T.K., M. E. Sullivan, in 'Prog.Med. Chem.', Eds. G. P. Ellis, D. K. Luscomb, Elsevier Science Publishers, Amsterdam, 1992 pp. 65-108.

[28] R. A. Pearlstein, R. J. Vaz, J. Kang, X. L. Chen, M. Preobrazhenskaya, A. E. Shchekotikhin, A. M. Korolev, L. N. Lysenkova, O. V. Miroshnikova, J. Hendrix, D. Rampe, Bioorg. Med. Chem. Lett. 2003, 13, 1829.

[29] A. Cavalli, E. Poluzzi, F. De Ponti, M. Recanatini, J. Med. Chem. 2002, 45, 3844.

[30] M. C. Sanguinetti, J. S. Mitcheson, Trends Pharmacol. Sci. 2005, 26, 119 .

[31] J. S. Mitcheson, J. Chen, M. Lin, C. Culberson, M. C. Sanguinetti, Proc. Natl. Acad. Sci. USA 2000, 97, 12329.

[32] E. Ficker, W. Jarolimek, J. Kiehn, A. Baumann, A. M. Brown, Circ. Res. 1998, 82, 386.

[33] D. Fernandez, A. Ghanta, G. W. Kauffman, M. C. Sanguinetti, J. Biol. Chem. 2004, 279, 10120 .

[34] J. A. Sanchez-Chapula, R. A. Navarro-Polanco, C. Culberson, J. Chen, M. C. Sanguinetti, J. Biol. Chem. 2002, 26, 23587.

[35] U. Zachariae, F. Giordanetto, A. G. Leach, J. Med. Chem. 2009, 52, 4266.

[36] K. Kamiya, R. Niwa, M. Morishima, H. Honjo, M. C. Sanguinetti, J. Pharmacol. Sci. 2008, $108,301$. 